\title{
Reciprocal Time Relation of Noncolliding Brownian Motion with Drift
}

\author{
Makoto Katori *
}

15 June 2012

\begin{abstract}
We consider an $N$-particle system of noncolliding Brownian motion starting from $x_{1} \leq x_{2} \leq \cdots \leq x_{N}$ with drift coefficients $\nu_{j}, 1 \leq j \leq N$ satisfying $\nu_{1} \leq \nu_{2} \leq \cdots \leq \nu_{N}$. When all of the initial points are degenerated to be zero, $x_{j}=0,1 \leq j \leq N$, the equivalence is proved between a dilatation with factor $1 / t$ of this drifted process and the noncolliding Brownian motion starting from $\nu_{1} \leq \nu_{2} \leq \cdots \leq \nu_{N}$ without drift observed at reciprocal time $1 / t$, for arbitrary $t>0$. Using this reciprocal time relation, we study the determinantal property of the noncolliding Brownian motion with drift having finite and infinite numbers of particles.
\end{abstract}

Keywords Noncolliding Brownian motion · Drift transform · Doob's $h$-transform · Determinantal processes · Correlation kernels · Infinite particle systems - Reciprocal time relation

\section{Introduction}

For $N=2,3, \ldots$, we consider an unbounded domain of $\mathbb{R}^{N}$,

$$
\mathbb{W}_{N}=\left\{\boldsymbol{x}=\left(x_{1}, x_{2}, \ldots, x_{N}\right) \in \mathbb{R}^{N}: x_{1}<x_{2}<\cdots<x_{N}\right\}
$$

which is called the Weyl chamber of type $\mathrm{A}_{N-1}$ in the representation theory [9]. Let $\boldsymbol{B}^{\boldsymbol{x}}(t), t \geq 0$ be an $N$-dimensional standard Brownian motion starting from $\boldsymbol{x} \in \mathbb{W}_{N}$. We assume that the boundary $\partial \mathbb{W}_{N}=\left\{\boldsymbol{x} \in \overline{\mathbb{W}}_{N}: 1 \leq^{\exists} j \leq N-1\right.$, s.t. $\left.x_{j}=x_{j+1}\right\}$ is made of absorbing walls in the sense that, if $\boldsymbol{B}^{\boldsymbol{x}}(t)$ hits any point of $\partial \mathbb{W}_{N}$, the Brownian particle is immediately absorbed there and the Brownian motion is stopped. For such an absorbing Brownian motion in $\mathbb{W}_{N}$, the survival probability $\mathcal{N}_{N}(T, \boldsymbol{x})$ is defined as the probability at

* Department of Physics, Faculty of Science and Engineering, Chuo University, Kasuga, Bunkyo-ku, Tokyo 112-8551, Japan; e-mail: katori@phys.chuo-u.ac.jp 
time $T \geq 0$ such that $\boldsymbol{B}^{\boldsymbol{x}}(T), \boldsymbol{x} \in \mathbb{W}_{N}$ is not yet absorbed at $\partial \mathbb{W}_{N}$ and still moving in $\mathbb{W}_{N}$. We can show that [18] for any $\boldsymbol{x} \in \mathbb{W}_{N}$,

$$
\mathcal{N}_{N}(T, \boldsymbol{x}) \simeq c_{N} T^{-N(N-1) / 4} h_{N}(\boldsymbol{x}) \rightarrow 0 \quad \text { as } \quad T \rightarrow \infty,
$$

where $c_{N}=\pi^{-N / 2} \prod_{j=1}^{N} \Gamma(j / 2) / \Gamma(j)$ with the Gamma function $\Gamma$ and

$$
h_{N}(\boldsymbol{x})=\operatorname{det}_{1 \leq j, k \leq N}\left[x_{j}^{k-1}\right]=\prod_{1 \leq j<k \leq N}\left(x_{k}-x_{j}\right) .
$$

On the other hand, provided that

$$
\boldsymbol{\nu}=\left(\nu_{1}, \nu_{2}, \ldots, \nu_{N}\right) \in \mathbb{W}_{N}
$$

the absorbing Brownian motion in $\mathbb{W}_{N}$ with drift $\boldsymbol{\nu}$,

$$
\widehat{\boldsymbol{B}}^{\boldsymbol{x}}(t)=\boldsymbol{B}^{\boldsymbol{x}}(t)+\boldsymbol{\nu} t, \quad t \in[0, \infty),
$$

starting from $\boldsymbol{x} \in \mathbb{W}_{N}$ has a positive probability to survive forever. If we regard the $j$ th component of (1.5) as position of a Brownian motion in $\mathbb{R}, 1 \leq j \leq N$, (1.5) gives a configuration at time $t$ of an $N$-particle system of one-dimensional standard Brownian motions. While $\widehat{\boldsymbol{B}}^{\boldsymbol{x}}(t) \in \mathbb{W}_{N}$, any collision of particles does not occur among $N$ particles, and only when $\widehat{\boldsymbol{B}}_{\boldsymbol{\nu}}^{\boldsymbol{x}}(t) \in \partial \mathbb{W}_{N}$, particle collision occurs. If $\boldsymbol{x} \in \mathbb{W}_{N}$ and (1.4) is satisfied, that is, the ordering of initial positions coincides with that of values of drift coefficients; $x_{j}<x_{j+1}, \nu_{j}<\nu_{j+1}, 1 \leq j \leq N-1$, the probability to avoid any collision of particles $\mathcal{N}_{N}^{\boldsymbol{\nu}}(T, \boldsymbol{x})$ is positive for all $T \geq 0$. As a matter of fact, Biane, Bougerol and O'Connell [3] gave the following determinantal expression for the long-term limit,

$$
\lim _{T \rightarrow \infty} \mathcal{N}_{N}^{\boldsymbol{\nu}}(T, \boldsymbol{x})=e^{-\boldsymbol{\nu} \cdot \boldsymbol{x}} \operatorname{det}_{1 \leq j, k \leq N}\left[e^{\nu_{j} x_{k}}\right]
$$

Conditionally on staying in $\mathbb{W}_{N}$ forever, we call the absorbing Brownian motion in $\mathbb{W}_{N}$ the noncolliding Brownian motion $[2,10,20]$. Without drift, $\boldsymbol{\nu}=0$, its transition probability density from $\boldsymbol{x} \in \mathbb{W}_{N}$ to $\boldsymbol{y} \in \mathbb{W}_{N}$ in time duration $0 \leq t<\infty$ is given by [18]

$$
\begin{aligned}
p_{N}(t, \boldsymbol{y} \mid \boldsymbol{x}) & =\lim _{T \rightarrow \infty} \frac{\mathcal{N}_{N}(T-t, \boldsymbol{y})}{\mathcal{N}_{N}(T, \boldsymbol{x})} q_{N}(t, \boldsymbol{y} \mid \boldsymbol{x}) \\
& =\frac{h_{N}(\boldsymbol{y})}{h_{N}(\boldsymbol{x})} q_{N}(t, \boldsymbol{y} \mid \boldsymbol{x}),
\end{aligned}
$$

where (1.2) has been used and $q_{N}$ is the transition probability density of the absorbing Brownian motion in $\mathbb{W}_{N}$ expressed by the Karlin-McGregor determinant [14]

$$
q_{N}(t, \boldsymbol{y} \mid \boldsymbol{x})=\operatorname{det}_{1 \leq j, k \leq N}\left[p\left(t, y_{j} \mid x_{k}\right)\right]
$$


Here $p$ is the transition probability density of the one-dimensional standard Brownian motion (i.e. the probability density of the Gaussian distribution with variance $t$ ),

$$
p(t, y \mid x)=\frac{1}{\sqrt{2 \pi t}} e^{-(y-x)^{2} / 2 t} .
$$

Equation (1.7) shows the fact that the noncolliding Brownian motion is Doob's $h$-transform by the Vandermonde determinant (the product of differences) (1.3) of the absorbing Brownian motion in $\mathbb{W}_{N}[2,10,24]$.

Remark 1 An important fact is that (1.7) with (1.8) satisfies the partial differential equation

$$
\left[\frac{\partial}{\partial t}-\left(\frac{1}{2} \Delta+\nabla \log h_{N}(\boldsymbol{x}) \cdot \nabla\right)\right] p_{N}(t, \boldsymbol{y} \mid \boldsymbol{x})=0
$$

with the initial condition $p_{N}(0, \boldsymbol{y} \mid \boldsymbol{x})=\delta(\boldsymbol{x}-\boldsymbol{y}) \equiv \prod_{j=1}^{N} \delta\left(x_{j}-y_{j}\right)$, where $\Delta=\sum_{j=1}^{N} \partial^{2} / \partial x_{j}^{2}$ and $\nabla=\left(\partial / \partial x_{1}, \ldots, \partial / \partial x_{N}\right)$, which is identified with the backward Kolmogorov equation of Dyson's Brownian motion model with parameter $\beta=2[7,10,19]$. That is, the noncolliding Brownian motion is equivalent to the eigenvalue process of Hermitian-matrix-valued Brownian motion, which has been extensively studied as a typical example of log-gas system in the random matrix theory $[29,8,24]$.

When $\boldsymbol{\nu} \neq 0$ the transition probability density of the noncolliding Brownian motion will be given by

$$
p_{N}^{\nu}(t, \boldsymbol{y} \mid \boldsymbol{x})=\lim _{T \rightarrow \infty} \frac{\mathcal{N}_{N}^{\boldsymbol{\nu}}(T-t, \boldsymbol{y})}{\mathcal{N}_{N}^{\boldsymbol{\nu}}(T, \boldsymbol{x})} q_{N}^{\boldsymbol{\nu}}(t, \boldsymbol{y} \mid \boldsymbol{x}), \quad \boldsymbol{x}, \boldsymbol{y} \in \mathbb{W}_{N}, \quad t \in[0, \infty)
$$

with the drift transform of (1.8),

$$
q_{N}^{\nu}(t, \boldsymbol{y} \mid \boldsymbol{x})=\exp \left\{-\frac{t}{2}|\boldsymbol{\nu}|^{2}+\boldsymbol{\nu} \cdot(\boldsymbol{y}-\boldsymbol{x})\right\} q_{N}(t, \boldsymbol{y} \mid \boldsymbol{x}) .
$$

If (1.4) is satisfied, by (1.6) Biane, Bougerol and O'Connell derived the expression [3]

$$
p_{N}^{\nu}(t, \boldsymbol{y} \mid \boldsymbol{x})=e^{-t|\boldsymbol{\nu}|^{2} / 2} \frac{\operatorname{det}_{1 \leq j, k \leq N}\left[e^{\nu_{j} y_{k}}\right]}{\operatorname{det}_{1 \leq j, k \leq N}\left[e^{\nu_{j} x_{k}}\right]} q_{N}(t, \boldsymbol{y} \mid \boldsymbol{x}), \quad \boldsymbol{x}, \boldsymbol{y}, \boldsymbol{\nu} \in \mathbb{W}_{N}, \quad t \in[0, \infty) .
$$

We note that even when some of $\nu_{j}$ 's in $\boldsymbol{\nu}=\left(\nu_{1}, \ldots, \nu_{N}\right)$ coincide, the ratio of determinants $\operatorname{det}_{1 \leq j, k \leq N}\left[e^{\nu_{j} y_{k}}\right] / \operatorname{det}_{1 \leq j, k \leq N}\left[e^{\nu_{j} x_{k}}\right]$ can be interpreted using l'Hôpital's rule. Then the domain of $\boldsymbol{\nu}$ is extended from (1.4) to

$$
\boldsymbol{\nu} \in \overline{\mathbb{W}}_{N}=\left\{\boldsymbol{x} \in \mathbb{R}^{N}: x_{1} \leq x_{2} \leq \cdots \leq x_{N}\right\} .
$$

In particular, if we take the limit $\nu_{j} \rightarrow 0,1 \leq j \leq N,(1.13)$ is reduced to (1.7) (see Appendix A). Similarly, the initial configuration $\boldsymbol{x}$ of (1.13) can be also extended to any element of $\overline{\mathbb{W}}_{N}$. 
Remark 2 Recently O'Connell [30] introduced an interacting diffusive particle system, which is regarded as a multivariate extension of a one-dimensional diffusion studied by Matsumoto and Yor $[27,28]$. Let $\psi_{\boldsymbol{\nu}}^{(N)}(\boldsymbol{x}), \boldsymbol{x} \in \mathbb{R}^{N}, \boldsymbol{\nu} \in \mathbb{C}^{N}$ be the class-one Whittaker function $[1,30,15,16,6,31,5]$, whose Givental integral representation is given by

$$
\begin{aligned}
\psi_{\boldsymbol{\nu}}^{(N)}(\boldsymbol{x})= & \int_{\Gamma_{N}(\boldsymbol{x})} \exp \left[\sum_{j=1}^{N} \nu_{j}\left(\sum_{k=1}^{j} T_{j, k}-\sum_{k=1}^{j-1} T_{j-1, k}\right)\right. \\
& \left.-\sum_{j=1}^{N-1} \sum_{k=1}^{j}\left\{e^{-\left(T_{j, k}-T_{j+1, k}\right)}+e^{-\left(T_{j+1, k+1}-T_{j, k}\right)}\right\}\right] d \boldsymbol{T},
\end{aligned}
$$

where the integral is performed over the space of all real lower triangular arrays with size $N, \boldsymbol{T}=\left(T_{j, k}, 1 \leq k \leq j \leq N\right)$ conditioned $T_{N, k}=x_{k}, 1 \leq k \leq N$. The transition probability density $p_{N}^{\boldsymbol{\nu}, a}$ of the O'Connell process is a unique solution of a "geometric lifting with parameter $a>0$ " $[4,16]$ of $(1.10)$,

$$
\left[\frac{\partial}{\partial t}-\left(\frac{1}{2} \Delta+\nabla \log \psi_{\boldsymbol{\nu}}^{(N)}(\boldsymbol{x} / a) \cdot \nabla\right)\right] P_{N}^{\boldsymbol{\nu}, a}(t, \boldsymbol{y} \mid \boldsymbol{x})=0
$$

with the initial condition $P_{N}^{\boldsymbol{\nu}, a}(0, \boldsymbol{y} \mid \boldsymbol{x})=\delta(\boldsymbol{x}-\boldsymbol{y})$, where the $\log$-potential $\log h_{N}(\boldsymbol{x})$ in (1.10) is replaced by $\log \psi_{\boldsymbol{\nu}}^{(N)}(\boldsymbol{x} / a)$. The solution is given by

$$
P_{N}^{\boldsymbol{\nu}, a}(t, \boldsymbol{y} \mid \boldsymbol{x})=e^{-t|\boldsymbol{\nu}|^{2} / 2 a^{2}} \frac{\psi_{\boldsymbol{\nu}}^{(N)}(\boldsymbol{y} / a)}{\psi_{\boldsymbol{\nu}}^{(N)}(\boldsymbol{x} / a)} Q_{N}^{a}(t, \boldsymbol{y} \mid \boldsymbol{x})
$$

with

$$
Q_{N}^{a}(t, \boldsymbol{y} \mid \boldsymbol{x})=\int_{\mathbb{R}^{N}} e^{-t|\boldsymbol{k}|^{2} / 2} \psi_{i a}^{(N)}(\boldsymbol{x} / a) \psi_{-i a}^{(N)}(\boldsymbol{y} / a) s_{N}(a \boldsymbol{k}) d \boldsymbol{k},
$$

where $s_{N}(\boldsymbol{\mu})$ is the density function of the Sklyanin measure

$$
s_{N}(\boldsymbol{\mu})=\frac{1}{(2 \pi)^{N} N !} \prod_{1 \leq j<\ell \leq N}\left|\Gamma\left(i\left(\mu_{\ell}-\mu_{j}\right)\right)\right|^{-2} .
$$

We can show (see Appendix B) that (1.13) is regarded as the "tropical analogue" of the transition probability density of the O'Connell process in the sense that

$$
p_{N}^{\nu}(t, \boldsymbol{y} \mid \boldsymbol{x})=\lim _{a \rightarrow 0} P_{N}^{a \boldsymbol{\nu}, a}(t, \boldsymbol{y} \mid \boldsymbol{x}), \quad \boldsymbol{x}, \boldsymbol{\nu} \in \overline{\mathbb{W}}_{N}, \boldsymbol{y} \in \mathbb{W}, t \in[0, \infty) .
$$

In the present paper, we study the noncolliding Brownian motion with drift satisfying (1.14) under the special initial condition such that all particles are put at the origin, $\mathbf{0}=$ $(0,0, \ldots, 0)$. By taking the limit $|\boldsymbol{x}| \rightarrow 0,(1.13)$ gives

$$
\begin{aligned}
p_{N}^{\boldsymbol{\nu}}(t, \boldsymbol{y} \mid \mathbf{0}) & =\lim _{|\boldsymbol{x}| \rightarrow 0} p_{N}^{\nu}(t, \boldsymbol{y} \mid \boldsymbol{x}) \\
& =t^{-N} \frac{h_{N}(\boldsymbol{y} / t)}{h_{N}(\boldsymbol{\nu})} q_{N}\left(t^{-1}, \boldsymbol{y} / t \mid \boldsymbol{\nu}\right),
\end{aligned}
$$


where $\boldsymbol{y} / t \equiv\left(y_{1} / t, \ldots, y_{N} / t\right)$ (see Appendix A). Then comparing with (1.7) we obtain the equality

$$
p_{N}^{\nu}(t, \boldsymbol{y} \mid \mathbf{0}) d \boldsymbol{y}=p_{N}\left(t^{-1}, \boldsymbol{y} / t \mid \boldsymbol{\nu}\right) d(\boldsymbol{y} / t), \quad \boldsymbol{y} \in \mathbb{W}_{N}, \quad \boldsymbol{\nu} \in \overline{\mathbb{W}}_{N}, \quad t \in[0, \infty),
$$

where $d(\boldsymbol{y} / t)=\prod_{j=1}^{N} d y_{j} / t=t^{-N} d \boldsymbol{y}$. It implies the equivalence between a dilatation by factor $1 / t$ of the noncolliding Brownian motion with drift $\boldsymbol{\nu} \in \overline{\mathbb{W}}_{N}$ and the process without drift observed at reciprocal time $1 / t$ (the reciprocal time relation). Essentially the same equation with (1.21) was given by Jones and O'Connell (Proposition 2.3 in [12]) and the equivalence (1.22) in more generality was discussed at a special time $t=1$. In the present paper, we extend their result to the level of processes, in which the time change $t \rightarrow 1 / t$ is associated. We note that, for the case $N=1$, the statement is just a rewriting of a well-known drift transformation (see, for instance, [13]). Here we discuss the reciprocal time relation in interacting particle systems.

In an earlier paper [22], the noncolliding Brownian motion with $\boldsymbol{\nu}=0$ (the Dyson model) with finite and infinite numbers of particles were systematically studied. If the number of particles is finite $N<\infty$ and an initial configuration is deterministic $\xi_{N}=\sum_{j=1}^{N} \delta_{x_{j}}$, then it is proved that the process is determinantal and the multitime correlation kernel is explicitly given as a functional of $\xi_{N}$ (Proposition 2.1 of [22]), which is denoted by $\mathbb{K}^{\xi_{N}}$. By the equality (1.22), we can conclude that at arbitrary time $t \in[0, \infty)$, the particle distribution of the noncolliding Brownian motion with drift $\boldsymbol{\nu} \in \mathbb{W}_{N}$ starting from $\mathbf{0}$ is a determinantal point process $[35,34]$ with the spatial correlation kernel $\mathbb{K}^{\nu_{N}}\left(t^{-1}, x / t ; t^{-1}, y / t\right) \times(1 / t)$ with $\nu_{N} \equiv \sum_{j=1}^{N} \delta_{\nu_{j}}$. This spatial correlation kernel is written as

$$
\mathbf{K}_{\nu_{N}}(t, x ; t, y)=t \sum_{j=1}^{N} p\left(t, y \mid t \nu_{j}\right) \int_{\mathbb{R}} d \mu^{\prime} p\left(t,-i x \mid t \mu^{\prime}\right) \prod_{1 \leq k \leq N, k \neq j}\left(1-\frac{i \mu^{\prime}-\nu_{j}}{\nu_{k}-\nu_{j}}\right),
$$

$(x, y) \in \mathbb{R}^{2}, t \in[0, \infty)$, where $i=\sqrt{-1}$ and $p$ is given by (1.9).

In this paper, we prove the reciprocal time relation of the noncolliding Brownian motion with drift (Theorem 2.1). Then by a combination of this theorem and Proposition 2.1 in [22], we conclude that if the number of particles is finite the noncolliding Brownian motion starting from $\mathbf{0}$ is determinantal for any $\boldsymbol{\nu} \in \overline{\mathbb{W}}_{N}$ and the explicit form of multitime correlation kernel $\mathbf{K}_{\nu_{N}}(s, \cdot ; t, \cdot),(s, t) \in[0, \infty)^{2}$ is determined (Proposition $2.2(\mathrm{i})$ ). There if $\boldsymbol{\nu} \in \mathbb{W}_{N}$, the expression $\mathbf{K}_{\nu_{N}}$ is simplified (Proposition 2.2 (ii)). Then by applying the theory of the Dyson model with an infinite number of particles [21, 22, 23, 25], infinite particle limits are discussed (Proposition 2.3, Corollary 2.4, and Corollary 2.5).

We would like to put emphasis on the fact that the reciprocal time relation will play an important role in understanding some of recent results for the exactly solvable interacting particle systems. See a comment given below Corollary 4.2 in [30] with [11] and [17].

The paper is organized as follows. In Sect.2 preliminaries and main results are given. Section 3 is devoted to proofs of results. Appendices A and B are prepared for explaining the facts used in Sect.1. 


\section{Preliminaries and Main Results}

For a finite number of particles $N<\infty$, each fixed configuration $\boldsymbol{x}=\left(x_{1}, x_{2}, \ldots, x_{N}\right) \in \mathbb{R}^{N}$ is identified with a finite summation of delta measures, $\xi_{N}(\cdot)=\sum_{j=1}^{N} \delta_{x_{j}}(\cdot)$, if particles are indistinguishable. For example, the configuration that all $N$ particles are put at the origin, which is written as $\mathbf{0}$ in $(1.21)$, is identified with $N \delta_{0}(\cdot)$. In order to discuss configurations with $N=\infty$ also, we consider the space of nonnegative integer-valued Radon measures on $\mathbb{R}$ denoted by $\mathfrak{M}$. Any element $\xi$ of $\mathfrak{M}$ can be represented as $\xi(\cdot)=\sum_{j \in \Lambda} \delta_{x_{j}}(\cdot)$ with a sequence of points in $\mathbb{R}, \boldsymbol{x}=\left(x_{j}\right)_{j \in \Lambda}$, where the index set $\Lambda$ is a finite set, the set of natural numbers $\mathbb{N}=\{1,2, \ldots\}$ or that of integers $\mathbb{Z}=\{\ldots,-1,0,1,2, \ldots\}$, and $\xi(K)=\sharp\{j \in$ $\left.\Lambda: x_{j} \in K\right\}<\infty$ for any compact subset $K \subset \mathbb{R}$. We call an element $\xi$ of $\mathfrak{M}$ a unlabeled configuration, and a sequence $\boldsymbol{x}$ a labeled configuration. For $\xi(\cdot)=\sum_{j \in \Lambda} \delta_{x_{j}}(\cdot) \in \mathfrak{M}$ and $A \subset \mathbb{R}$, we write the restriction of $\xi$ in $A$ as $(\xi \cap A)(\cdot)=\sum_{j \in \Lambda: x_{j} \in A} \delta_{x_{j}}(\cdot)$. The dilatation of $\xi(\cdot)$ with factor $c>0$ is written as $c \circ \xi(\cdot)=\sum_{j \in \Lambda} \delta_{c x_{j}}(\cdot)$.

First we assume that the initial configuration $\xi_{N}$ has a finite number of particles, $\xi_{N}(\mathbb{R})=$ $N \in \mathbb{N}$. We consider an element of $\mathfrak{M}$ corresponding to the drift coefficients $\boldsymbol{\nu}=\left(\nu_{1}, \ldots, \nu_{N}\right) \in$ $\overline{\mathbb{W}}_{N}$ and write it as $\nu_{N}(\cdot)=\sum_{j=1}^{N} \delta_{\nu_{j}}(\cdot)$. For $M \in \mathbb{N}$, the multitime joint probability density for arbitrary $M$ sequence of times $0<t_{1}<t_{2}<\cdots<t_{M}<\infty$ of the noncolliding Brownian motion with drift $\boldsymbol{\nu} \in \overline{\mathbb{W}}_{N}$ is given by

$$
\begin{aligned}
& p_{\nu_{N}}^{\xi_{N}}\left(t_{1}, \xi^{(1)} ; \cdots: t_{M}, \xi^{(M)}\right) \\
& =\prod_{m=1}^{M-1} p_{N}^{\nu}\left(t_{m+1}-t_{m} ; \boldsymbol{x}^{(m+1)} \mid \boldsymbol{x}^{(m)}\right) p_{N}^{\nu}\left(t_{1}, \boldsymbol{x}^{(1)} \mid \boldsymbol{x}\right) \\
& =e^{-t_{M}|\boldsymbol{\nu}|^{2} / 2} \operatorname{det}_{1 \leq j, k \leq N}\left[e^{\nu_{j} x_{k}^{(M)}}\right] \prod_{m=1}^{M-1} q_{N}\left(t_{m+1}-t_{m}, \boldsymbol{x}^{(m+1)} \mid \boldsymbol{x}^{(m)}\right) \frac{q_{N}\left(t_{1}, \boldsymbol{x}^{(1)} \mid \boldsymbol{x}\right)}{\operatorname{det}_{1 \leq j, k \leq N}\left[e^{\nu_{j} x_{k}}\right]}
\end{aligned}
$$

with $\xi^{(m)}=\sum_{j=1}^{N} \delta_{x_{j}^{(m)}}, \boldsymbol{x}^{(m)}=\left(x_{1}^{(m)}, \ldots, x_{N}^{(m)}\right) \in \mathbb{W}_{N}, 1 \leq m \leq M, \boldsymbol{x}=\left(x_{1}, \ldots, x_{N}\right) \in \overline{\mathbb{W}}_{N}$, where (1.13) has been used. We can take the limit $\boldsymbol{\nu} \rightarrow \mathbf{0}$ of (2.1) (using (A.1) in Appendix A) and obtain the following expression for the multitime joint probability density of the noncolliding Brownian motion without drift [20],

$$
\begin{aligned}
& p^{\xi_{N}}\left(t_{1}, \xi^{(1)} ; \cdots ; t_{M}, \xi^{(M)}\right) \\
& =h_{N}\left(\boldsymbol{x}^{(M)}\right) \prod_{m=1}^{M-1} q_{N}\left(t_{m+1}-t_{m} ; \boldsymbol{x}^{(m+1)} \mid \boldsymbol{x}^{(m)}\right) \frac{q_{N}\left(t_{1}, \boldsymbol{x}^{(1)} \mid \boldsymbol{x}\right)}{h_{N}(\boldsymbol{x})} .
\end{aligned}
$$

The stochastic differential equation of the noncolliding Brownian motion with drift $\boldsymbol{\nu}$, $\boldsymbol{X}(t)=\left(X_{1}(t), \ldots, X_{N}(t)\right), t \in[0, \infty)$ is given by

$$
d X_{j}(t)=d B_{j}(t)+\nu_{j} t+\sum_{1 \leq k \leq N, k \neq j} \frac{d t}{X_{j}(t)-X_{k}(t)}, \quad 1 \leq j \leq N \in \mathbb{N}, \quad t \in[0, \infty),
$$

where $\left\{B_{j}(t)\right\}_{j=1}^{N}$ are independent one-dimensional standard Brownian motions starting from 0 . Let $\Xi(t, \cdot)=\sum_{j=1}^{N} \delta_{X_{j}(t)}(\cdot), t \in[0, \infty)$ and the probability measure of the process starting 
from $\xi_{N} \in \mathfrak{M}$ with drift $\nu_{N}$ be denoted by $\underset{\nu_{N}}{\xi_{N}}$. The finite dimensional distribution of $\mathbb{P}_{\nu_{N}}^{\xi_{N}}$ is given by (2.1). For the noncolliding Brownian motion without drift starting from $\xi_{N}$, the probability measure is simply denoted by $\mathbb{P}^{\xi_{N}}$, whose finite dimensional distribution is given by (2.2). In general, two processes having the same state space are said to be equivalent, if they have the same finite-dimensional distributions, that is, if for any finite sequence of times $0<t_{1}<\cdots<t_{M}<\infty, M \in \mathbb{N}$, the multitime joint probability density functions coincide with each other [32]. Here we use a symbol $\stackrel{\text { (law), }}{=}$ to express equivalence of processes.

The main theorem of the present paper is the following.

Theorem 2.1 If $\nu_{N} \in \mathfrak{M}$ with $\nu_{N}(\mathbb{R})=N \in \mathbb{N}$, the following equivalence is established,

$$
\left(\frac{1}{t} \circ \Xi(t), t \in[0, \infty), \mathbb{P}_{\nu_{N}}^{N \delta_{0}}\right) \stackrel{(\text { law })}{=}\left(\Xi\left(\frac{1}{t}\right), t \in[0, \infty), \mathbb{P}^{\nu_{N}}\right) .
$$

We call this equivalence between a dilatation with factor $1 / t$ of the drifted process and the process without drift observed at reciprocal time $1 / t$ the reciprocal time relation in this paper.

For $\boldsymbol{x}^{(m)}=\left(x_{1}^{(m)}, \ldots, x_{N}^{(m)}\right) \in \mathbb{W}_{N}$ and $N^{\prime} \in\{1,2, \ldots, N\}$, we put $\boldsymbol{x}_{N^{\prime}}^{(m)}=\left(x_{1}^{(m)}, \ldots, x_{N^{\prime}}^{(m)}\right) \in$ $\mathbb{W}_{N^{\prime}}, 1 \leq m \leq M$. For a sequence $\left(N_{m}\right)_{m=1}^{M}$ of positive integers less than or equal to $N$, we define the $\left(N_{1}, \ldots, N_{M}\right)$-multitime correlation function by

$$
\begin{aligned}
& \rho_{\nu_{N}}^{N \delta_{0}}\left(t_{1}, \boldsymbol{x}_{N_{1}}^{(1)} ; \ldots ; t_{M}, \boldsymbol{x}_{N_{M}}^{(M)}\right) \\
& =\int_{\prod_{m=1}^{M} \mathbb{R}^{N-N_{m}}} \prod_{m=1}^{M} \prod_{j=N_{m}+1}^{N} d x_{j}^{(m)} p_{\nu_{N}}^{N \delta_{0}}\left(t_{1}, \xi_{N}^{(1)} ; \ldots ; t_{M}, \xi_{N}^{(M)}\right) \prod_{m=1}^{M} \frac{1}{\left(N-N_{m}\right) !}
\end{aligned}
$$

which is symmetric in the sense that $\rho_{\nu_{N}}^{N \delta_{0}}\left(\ldots ; t_{m}, \sigma\left(\boldsymbol{x}_{N_{m}}^{(m)}\right) ; \ldots\right)=\rho_{\nu_{N}}^{N \delta_{0}}\left(\ldots ; t_{m}, \boldsymbol{x}_{N_{m}}^{(m)} ; \ldots\right)$ with $\sigma\left(\boldsymbol{x}_{N_{m}}^{(m)}\right) \equiv\left(x_{\sigma(1)}^{(m)}, \ldots, x_{\sigma\left(N_{m}\right)}^{(m)}\right)$ for any permutation $\sigma \in \mathcal{S}_{N_{m}}, 1 \leq \forall m \leq M$.

If there is a function $\mathbf{K}(s, x ; t, y)$, which is continuous with respect to $(x, y) \in \mathbb{R}^{2}$ for any fixed $(s, t) \in[0, \infty)^{2}$, such that it determines the finite dimensional distributions of the process by giving determinantal expressions to multitime correlation functions $\rho$ as

$$
\rho\left(t_{1}, \boldsymbol{x}_{N_{1}}^{(1)} ; \ldots ; t_{M}, \boldsymbol{x}_{N_{M}}^{(M)}\right)=\operatorname{det}_{\substack{1 \leq j \leq N_{m}, 1 \leq k \leq N_{n} \\ 1 \leq m, n \leq M}}\left[\mathbf{K}\left(t_{m}, x_{j}^{(m)} ; t_{n}, x_{k}^{(n)}\right)\right]
$$

for any integer $M \geq 1$, any sequence $\left(N_{m}\right)_{m=1}^{M}$ of positive integers, and any time sequence $0<t_{1}<\cdots<t_{M}<\infty$, then the process is said to be determinantal with the correlation kernel $\mathbf{K}[22]$.

We use the convention such that

$$
\prod_{x \in \xi} f(x)=\exp \left\{\int_{\mathbb{R}} \xi(d x) \log f(x)\right\}=\prod_{x \in \operatorname{supp} \xi} f(x)^{\xi(\{x\})}
$$

for $\xi \in \mathfrak{M}$ and a function $f$ on $\mathbb{R}$, where supp $\xi=\{x \in \mathbb{R}: \xi(\{x\})>0\}$. Let $\mathbf{1}(\omega)$ be the indicator function of a condition $\omega ; \mathbf{1}(\omega)=1$ if $\omega$ is satisfied and $\mathbf{1}(\omega)=0$ otherwise. 
We consider a set of configurations with no multiple points,

$$
\mathfrak{M}_{0}=\{\xi \in \mathfrak{M}: \xi(\{x\}) \leq 1 \text { for any } x \in \mathbb{R}\} .
$$

Since any element $\xi$ of $\mathfrak{M}_{0}$ is determined uniquely by its support, it is identified with a countable subset $\left\{x_{j}\right\}_{j \in \Lambda}$ of $\mathbb{R}$. For $\xi_{N} \in \mathfrak{M}_{0}, a \in \mathbb{C}$, we introduce an entire function of $z \in \mathbb{C}$

$$
\Phi\left(\xi_{N}, a, z\right)=\prod_{u \in \xi_{N} \cap\{a\}^{\mathrm{c}}}\left(1-\frac{z-a}{u-a}\right),
$$

where $A^{\mathrm{c}}$ denotes a complementary set of $A$. The zero set of $\Phi\left(\xi_{N}, a, z\right)$ is $\operatorname{supp}\left(\xi_{N} \cap\{a\}^{\mathrm{c}}\right)$ (see, for instance, [26]). Then, as an application of Proposition 2.1 in [22], Theorem 2.1 gives the following.

Proposition 2.2 (i) The noncolliding Brownian motion with drift $\nu_{N} \in \mathfrak{M}$ with $\nu_{N}(\mathbb{R})=$ $N \in \mathbb{N},\left(\Xi(t), t \in[0, \infty), \mathbb{P}_{\nu_{N}}^{N \delta_{0}}\right)$, is determinantal with the correlation kernel

$$
\begin{aligned}
\mathbf{K}_{\nu_{N}}(s, x ; t, y)= & \frac{\sqrt{s t}}{2 \pi i} \oint_{\Gamma\left(\nu_{N}\right)} d \mu p(s, x \mid s \mu) \int_{\mathbb{R}} d \mu^{\prime} p\left(t,-i y \mid t \mu^{\prime}\right) \frac{1}{i \mu^{\prime}-\mu} \prod_{u \in \nu_{N}}\left(1-\frac{i \mu^{\prime}-\mu}{u-\mu}\right) \\
& -\mathbf{1}(s<t) p\left(t-s, \sqrt{\frac{s}{t}} y \mid \sqrt{\frac{t}{s}} x\right), \quad(s, t) \in[0, \infty)^{2},(x, y) \in \mathbb{R}^{2}, \quad
\end{aligned}
$$

where $\Gamma\left(\nu_{N}\right)$ is a closed contour on the complex plane $\mathbb{C}$ encircling the points in supp $\nu_{N}$ on the real line $\mathbb{R}$ once in the positive direction.

(ii) If $\nu_{N} \in \mathfrak{M}_{0}$ with $\nu_{N}(\mathbb{R})=N \in \mathbb{N}$, the correlation kernel (2.9) is simplified as

$$
\begin{gathered}
\mathbf{K}_{\nu_{N}}(s, x ; t, y)=\sqrt{s t} \sum_{j=1}^{N} p\left(s, x \mid s \nu_{j}\right) \int_{\mathbb{R}} d \mu^{\prime} p\left(t,-i y \mid t \mu^{\prime}\right) \Phi\left(\nu_{N}, \nu_{j}, i \mu^{\prime}\right) \\
\quad-\mathbf{1}(s<t) p\left(t-s, \sqrt{\frac{s}{t}} y \mid \sqrt{\frac{t}{s}}\right), \quad(s, t) \in[0, \infty)^{2},(x, y) \in \mathbb{R}^{2} .
\end{gathered}
$$

Note that, if $\nu_{N} \in \mathfrak{M}_{0}$,

$$
\Phi\left(\nu_{N}, \nu_{j}, i \mu^{\prime}\right)=\prod_{1 \leq k \leq N, k \neq j}\left(1-\frac{i \mu^{\prime}-\nu_{j}}{\nu_{k}-\nu_{j}}\right),
$$

and thus, when we set $s=t,(2.10)$ is reduced to be $(1.23)$.

Remark 3 The reciprocal time relation (2.4) will define a kind of duality between two processes $\left(\Xi(t), t \in[0, \infty), \mathbb{P}_{\nu}^{\xi}\right)$ and $\left(\Xi(1 / t), t \in[0, \infty), \mathbb{P}_{\xi}^{\nu}\right)$, where the initial configuration and drift coefficients are exchanged. A self-dual process, which satisfies the equality,

$$
\left(\frac{1}{t} \circ \Xi(t), t \in[0, \infty), \mathbb{P}^{N \delta_{0}}\right) \stackrel{(\text { law })}{=}\left(\Xi\left(\frac{1}{t}\right), t \in[0, \infty), \mathbb{P}^{N \delta_{0}}\right),
$$


$N \in \mathbb{N}$, is known as the determinantal process with the extended Hermite-function kernel (see, for example, Eq.(4.25) of [20], Section 5.1 of [24]).

For $\nu \in \mathfrak{M}$, when $\mathbf{K}_{\nu \cap[-L, L]}$ converges to a continuous function as $L \rightarrow \infty$, the limit is written as $\mathbf{K}_{\nu}$. If the probability measure $\mathbb{P}_{\nu \cap[-L, L]}$ of the process determined by $\mathbf{K}_{\nu \cap[-L, L]}$ converges to a probability measure $\mathbb{P}_{\nu}$ on $\mathfrak{M}^{[0, \infty)}$, which is determinantal with the correlation kernel $\mathbf{K}_{\nu}$, weakly in the sense of finite dimensional distributions as $L \rightarrow \infty$ in the vague topology, we say that the obtained process is well defined with the correlation kernel $\mathbf{K}_{\nu}$ $[21,22,23]$. The regularity of the sample paths can be discussed as [25]. In the case $\nu(\mathbb{R})=\infty$, the process obtained by this limit has an infinite number of particles with drift $\nu$.

In [22], two sets of configurations with finite and infinite numbers of particles were introduced and denoted by $\mathfrak{X}$ and $\mathfrak{Y}$. By the argument given there, the following statements can be proved for the noncolliding Brownian motion with drift $\nu$, which can be constructed from an infinite number of particles all starting from the origin. This infinite particle process will be denoted by $\left(\Xi(t), t \in[0, \infty), \mathbb{P}_{\nu}^{0}\right)$.

Proposition 2.3 (i) The infinite particle system of noncolliding Brownian motion with all particles starting from the origin, $\left(\Xi(t), t \in[0, \infty), \mathbb{P}_{\nu}^{0}\right)$, is well defined, if the drift coefficients $\nu \in \mathfrak{X} \cap \mathfrak{M}_{0}$. The correlation kernel is given by

$$
\begin{gathered}
\mathbf{K}_{\nu}(s, x ; t, y)=\sqrt{s t} \int_{\mathbb{R}} \nu(d \mu) p(s, x \mid s \mu) \int_{\mathbb{R}} d \mu^{\prime} p\left(t,-i y \mid t \mu^{\prime}\right) \Phi\left(\nu_{N}, \mu, i \mu^{\prime}\right) \\
-\mathbf{1}(s<t) p\left(s-t, \sqrt{\frac{s}{t}} y \mid \sqrt{\frac{t}{s}}\right), \quad(s, t) \in[0, \infty)^{2},(x, y) \in \mathbb{R}^{2} .
\end{gathered}
$$

(ii) If $\nu \in \mathfrak{Y},\left(\Xi(t), t \in[0, \infty), \mathbb{P}_{\nu}^{0}\right)$ is well defined. In particular, when $\nu \in \mathfrak{Y} \cap \mathfrak{M}_{0}$, the correlation kernel is given by (2.13).

As consequences of Theorem 2.1 and Proposition 2.3, we can conclude the following facts for the noncolliding Brownian motion with drift, whose number of particles is infinite. The reciprocal time relation (2.4) implies that, the long-term limit $t \rightarrow \infty$ of $\left((1 / t) \circ \Xi(t), \mathbb{P}_{\nu_{N}}^{N \delta_{0}}\right)$ is given by a $1 / t \rightarrow 0$ limit of $\left(\Xi(1 / t), \mathbb{P}^{\nu_{N}}\right)$, for any $N \in \mathbb{N}$. Since $\lim _{t \rightarrow \infty} \Xi(1 / t)=\nu_{N}$ in $\mathbb{P}^{\nu_{N}}$, we have the following.

Corollary 2.4 If $\nu \in \mathfrak{X}$ or $\nu \in \mathfrak{Y}$,

$$
\lim _{t \rightarrow \infty} \frac{1}{t} \circ \Xi(t)=\nu \quad \text { in } \mathbb{P}_{\nu}^{0}
$$

Let $\nu_{\mathbb{Z}}(\cdot)=\sum_{j \in \mathbb{Z}} \delta_{j}(\cdot)$. In [22], it is shown that $\nu_{\mathbb{Z}} \in \mathfrak{X} \cap \mathfrak{M}_{0}$ and the noncolliding Brownian motion without drift (the Dyson model) starting from $\nu_{\mathbb{Z}}$ is well defined. Moreover, it is proved that this infinite particle system shows a relaxation phenomenon to the equilibrium 
and spatially homogeneous determinantal process with the extended sine kernel with density 1 ,

$$
\begin{aligned}
\mathbf{K}_{\sin }(t-s, y-x) & =\frac{1}{2 \pi} \int_{|k| \leq \pi} d k e^{k^{2}(t-s) / 2+i k(y-x)}-\mathbf{1}(s>t) p(s-t, x \mid y) \\
& = \begin{cases}\int_{0}^{1} d u e^{\pi^{2} u^{2}(t-s) / 2} \cos \{\pi u(y-x)\} & \text { if } t>s \\
\frac{\sin \pi(y-x)}{\pi(y-x)} & \text { if } t=s \\
-\int_{1}^{\infty} d u e^{\pi^{2} u^{2}(t-s) / 2} \cos \{\pi u(y-x)\} & \text { if } t<s,\end{cases}
\end{aligned}
$$

$(s, t) \in[0, \infty)^{2},(x, y) \in \mathbb{R}^{2}$. Let $\vartheta_{3}$ be a version of the Jacobi theta function defined by

$$
\vartheta_{3}(v, \tau)=\sum_{n \in \mathbb{Z}} e^{2 \pi i v n+\pi i \tau n^{2}} . \quad \Im \tau>0
$$

Then by the reciprocal time relation (2.4), we have the following.

Corollary 2.5 The noncolliding Brownian motion with an infinite number of particles, $\left(\Xi(t), t \in[0, \infty), \mathbb{P}_{\nu_{Z}}^{0}\right)$, is well defined with the correlation kernel

$$
\begin{gathered}
\mathbf{K}_{\nu_{\mathbb{Z}}}(s, x ; t, y)=\frac{1}{2 \pi} \int_{|w| \leq \pi / \sqrt{s t}} d w e^{w^{2}(t-s) / 2+i w(y \sqrt{s / t}-x \sqrt{t / s})} \vartheta_{3}\left(\frac{x}{s}-i w \sqrt{\frac{t}{s}}, \frac{2 \pi i}{s}\right), \\
(s, t) \in[0, \infty)^{2},(x, y) \in \mathbb{R}^{2} .
\end{gathered}
$$

This kernel has the following asymptotics,

$$
\lim _{u \rightarrow \infty} u \mathbf{K}_{\nu_{\mathbb{Z}}}\left(\frac{1}{u+s}, \frac{x}{u+s} ; \frac{1}{u+t}, \frac{y}{u+t}\right)=\mathbf{K}_{\sin }(t-s, y-x) .
$$




\section{Proofs of Theorems}

Proof of Theorem 2.1 By (1.13), (2.1) is written as

$$
\begin{aligned}
& p_{\nu_{N}}^{\xi_{N}}\left(t_{1}, \xi^{(1)} ; \cdots ; t_{M}, \xi^{(M)}\right) \\
& =e^{-t_{M}|\boldsymbol{\nu}|^{2} / 2} \frac{\operatorname{det}_{1 \leq j, k \leq N}\left[e^{\nu_{j} x_{k}^{(M)}}\right]}{\operatorname{det}_{1 \leq j, k \leq N}\left[e^{\nu_{j} x_{k}^{(1)}}\right]} e^{t_{1}|\boldsymbol{\nu}|^{2} / 2} \prod_{m=1}^{M-1} q_{N}\left(t_{m+1}-t_{m} ; \boldsymbol{x}^{(m+1)} \mid \boldsymbol{x}^{(m)}\right) p_{N}^{\boldsymbol{\nu}_{N}}\left(t_{1}, \boldsymbol{x}^{(1)} \mid \boldsymbol{x}\right) \\
& =\frac{\left(2 \pi / t_{M}\right)^{N / 2}}{\operatorname{det}_{1 \leq j, k \leq N}\left[e^{\nu_{j} x_{k}^{(1)}}\right]} q_{N}\left(t_{M}^{-1}, \boldsymbol{x}^{(M)} / t_{M} \mid \boldsymbol{\nu}\right) e^{\left|\boldsymbol{x}^{(M)}\right|^{2} / 2 t_{M}} e^{t_{1}|\boldsymbol{\nu}|^{2} / 2} \\
& \quad \times \prod_{m=1}^{M-1} q_{N}\left(t_{m+1}-t_{m}, \boldsymbol{x}^{(m+1)} \mid \boldsymbol{x}^{(m)}\right) p_{N}^{\boldsymbol{\nu}_{N}}\left(t_{1}, \boldsymbol{x}^{(1)} \mid \boldsymbol{x}\right) .
\end{aligned}
$$

We rewrite the equality (1.22) as

$$
\begin{aligned}
p_{N}^{\boldsymbol{\nu}_{N}}(t, \boldsymbol{y} \mid \mathbf{0}) d \boldsymbol{y} & =p_{N}\left(t^{-1}, \boldsymbol{y} / t \mid \boldsymbol{\nu}\right) d(\boldsymbol{y} / t) \\
& =\frac{h_{N}(\boldsymbol{y} / t)}{h_{N}(\boldsymbol{\nu})} e^{-t|\boldsymbol{\nu}|^{2} / 2} e^{-|\boldsymbol{y}|^{2} / 2 t} \frac{\operatorname{det}_{1 \leq j, k \leq N}\left[e^{\nu_{j} y_{k}}\right]}{(2 \pi t)^{N / 2}} d \boldsymbol{y} .
\end{aligned}
$$

Then, we can confirm that for $\xi_{N}=N \delta_{0},(3.1)$ gives

$$
\begin{aligned}
p_{\nu_{N}}^{N \delta_{0}}\left(t_{1}, \xi^{(1)}\right. & \left.; \cdots ; t_{M}, \xi^{(M)}\right)=\left(t_{M} t_{1}\right)^{-N / 2} \frac{q_{N}\left(t_{M}^{-1}, \boldsymbol{x}^{(M)} / t_{M} \mid \boldsymbol{\nu}\right)}{h_{N}(\boldsymbol{\nu})} \\
& \times e^{\left|\boldsymbol{x}^{(M)}\right|^{2} / 2 t_{M}} \prod_{m=1}^{M-1} q_{N}\left(t_{m+1}-t_{m}, \boldsymbol{x}^{(m+1)} \mid \boldsymbol{x}^{(m)}\right) e^{-\left|\boldsymbol{x}^{(1)}\right|^{2} / 2 t_{1}} h_{N}\left(\boldsymbol{x}^{(1)} / t_{1}\right) .
\end{aligned}
$$

We find that the following equalities hold,

$$
\begin{aligned}
& q_{N}\left(t_{m+1}-t_{m}, \boldsymbol{x}^{(m+1)} \mid \boldsymbol{x}^{(m)}\right) \\
& =\left(t_{m+1} t_{m}\right)^{-N / 2} e^{-\left|\boldsymbol{x}^{(m+1)}\right|^{2} / 2 t_{m+1}} q_{N}\left(t_{m}^{-1}-t_{m+1}^{-1}, \frac{\boldsymbol{x}^{(m)}}{t_{m}} \mid \frac{\boldsymbol{x}^{(m+1)}}{t_{m+1}}\right) e^{\left|\boldsymbol{x}^{(m)}\right|^{2} / 2 t_{m}}, \\
& 1 \leq m \leq M .
\end{aligned}
$$

Then (3.3) is equal to

$$
h_{N}\left(\boldsymbol{x}^{(1)} / t_{1}\right) \prod_{m=1}^{M-1} q_{N}\left(t_{m}^{-1}-t_{m+1}^{-1}, \frac{\boldsymbol{x}^{(m)}}{t_{m}} \mid \frac{\boldsymbol{x}^{(m+1)}}{t_{m+1}}\right) \frac{q_{N}\left(t_{M}^{-1}, \boldsymbol{x}^{(M)} / t_{M} \mid \boldsymbol{\nu}\right)}{h_{N}(\boldsymbol{\nu})} \prod_{m=1}^{M} t_{m}^{-N} .
$$

Comparing this result with (2.2), we obtain the equality

$$
\begin{aligned}
& p_{\nu_{N}}^{N \delta_{0}}\left(t_{1}, \xi^{(1)} ; \cdots ; t_{M}, \xi^{(M)}\right) \prod_{m=1}^{M} d \boldsymbol{x}^{(m)} \\
& \quad=p^{\nu_{N}}\left(t_{M}^{-1}, \frac{1}{t_{M}} \circ \xi^{(M)} ; \cdots ; t_{1}^{-1}, \frac{1}{t_{1}} \circ \xi^{(1)}\right) \prod_{m=1}^{M}\left(\frac{d \boldsymbol{x}^{(m)}}{t_{m}}\right) .
\end{aligned}
$$


Then the equivalence (2.4) is proved.

Proof of Proposition 2.2 (i) By combination of Theorem 2.1 and Proposition 2.1 in [22], the correlation kernel is given by

$$
\begin{aligned}
& \mathbf{K}_{\nu_{N}}(s, x ; t, y)=\mathbb{K}^{\nu_{N}}\left(s^{-1}, x / s ; t^{-1}, y / t\right) \frac{1}{\sqrt{s t}} \\
& =\frac{1}{2 \pi i} \oint_{\Gamma\left(\nu_{N}\right)} d \mu p\left(s^{-1}, x / s \mid \mu\right) \int_{\mathbb{R}} d \mu^{\prime} p\left(t^{-1},-i y / t \mid \mu^{\prime}\right) \frac{1}{i \mu^{\prime}-\mu} \prod_{u \in \nu_{N}}\left(1-\frac{i \mu^{\prime}-\mu}{u-\mu}\right) \frac{1}{\sqrt{s t}} \\
& \quad-\mathbf{1}\left(s^{-1}>t^{-1}\right) p\left(s^{-1}-t^{-1}, x / s \mid y / t\right) \frac{1}{\sqrt{s t}} .
\end{aligned}
$$

Here by the cyclic property of determinant, a factor $1 / \sqrt{s t}$ should be put on the correlation kernel for the transform of variables, $(x, y) \mapsto(x / s, y / t)$. By (1.9), we see

$$
\begin{aligned}
& p\left(s^{-1}, x / s \mid \mu\right)=s p(s, x \mid s \mu), \\
& p\left(t^{-1},-i y / t \mid \mu^{\prime}\right)=t p\left(t,-i y \mid t \mu^{\prime}\right),
\end{aligned}
$$

and

$$
\mathbf{1}\left(s^{-1}>t^{-1}\right) p\left(s^{-1}-t^{-1}, x / s \mid y / t\right)=\mathbf{1}(s<t) \sqrt{s t} p\left(t-s, \sqrt{\frac{s}{t}} y \mid \sqrt{\frac{t}{s}} x\right) .
$$

Then (2.9) is obtained.

(ii) By performing the Cauchy integral in (2.9) on the contour $\Gamma\left(\nu^{N}\right),(2.10)$ is obtained.

Proof of Corollary 2.4 By Theorem 2.1, for $u, s, t \in[0, \infty), x, y \in \mathbb{R}$,

$$
\mathbf{K}_{\nu}(u+s, u x ; u+t, u y)=\mathbb{K}^{\nu}\left(\frac{1}{u+s}, \frac{u x}{u+s} ; \frac{1}{u+t}, \frac{u y}{u+t}\right) \sqrt{\frac{u}{u+s} \frac{u}{u+t}} .
$$

And

$$
\begin{gathered}
\mathbb{K}^{\nu}\left(\frac{1}{u+t}, \frac{u x}{u+t} ; \frac{1}{u+t}, \frac{u y}{u+t}\right) \mathbb{K}^{\nu}\left(\frac{1}{u+s}, \frac{u y}{u+s} ; \frac{1}{u+t}, \frac{u x}{u+t}\right)\left(\frac{u}{u+t}\right) d x d y \\
\rightarrow \nu(d x) \mathbf{1}(x=y) \quad \text { as } u \rightarrow \infty \text { in the vague topology. }
\end{gathered}
$$

Then the statement is obtained.

Proof of Corollary 2.5 The expression (2.17) is readily obtained from the formula (1.5) in [22] following the reciprocal time relation (2.4). We can check that

$$
\begin{aligned}
\lim _{u \rightarrow \infty} u \mathbf{K}_{\nu_{\mathbb{Z}}}\left(\frac{1}{u+s}, \frac{x}{u+s} ; \frac{1}{u+t}, \frac{y}{u+t}\right) & =\lim _{u \rightarrow \infty} \mathbb{K}^{\nu_{Z}}(u+s, x ; u+t, y) \frac{u}{\sqrt{(u+s)(u+t)}} \\
& =\mathbf{K}_{\sin }(t-s, y-x) .
\end{aligned}
$$

Then the statement is concluded. 


\section{Appendices}

\section{A Asymptotics of determinants}

By the Schur function expansion, we can prove that $[19,20]$ for $\boldsymbol{b} \in \mathbb{C}^{N}$

$$
\operatorname{det}_{1 \leq j, k \leq N}\left[e^{a_{j} b_{k}}\right]=\frac{h_{N}(\boldsymbol{a}) h_{N}(\boldsymbol{b})}{\prod_{j=1}^{N} \Gamma(j)} \times\{1+\mathcal{O}(|\boldsymbol{a}|)\} \quad \text { as } \quad|\boldsymbol{a}| \rightarrow 0 .
$$

Then

$$
\lim _{|\boldsymbol{\nu}| \rightarrow 0} \frac{\operatorname{det}_{1 \leq j, k \leq N}\left[e^{\nu_{j} y_{k}}\right]}{\operatorname{det}_{1 \leq j, k \leq N}\left[e^{\nu_{j} x_{k}}\right]}=\frac{h_{N}(\boldsymbol{y})}{h_{N}(\boldsymbol{x})}
$$

which implies the fact that (1.7) is reduced from (1.13) by taking $\nu_{j} \rightarrow 0,1 \leq j \leq N$.

Similarly, we can see that

$$
\lim _{|\boldsymbol{x}| \rightarrow 0} \frac{\operatorname{det}_{1 \leq j, k \leq N}\left[e^{x_{j} y_{k} / t}\right]}{\operatorname{det}_{1 \leq j, k \leq N}\left[e^{\nu_{j} x_{k}}\right]}=\lim _{|\boldsymbol{x}| \rightarrow 0} \frac{h_{N}(\boldsymbol{x} / \sqrt{t}) h_{N}(\boldsymbol{y} / \sqrt{t})}{h_{N}(\boldsymbol{\nu}) h_{N}(\boldsymbol{x})}=\frac{h_{N}(\boldsymbol{y} / t)}{h_{N}(\boldsymbol{\nu})}
$$

Since $q_{N}(t, \boldsymbol{y} \mid \boldsymbol{x})$ given by (1.8) is equal to $(2 \pi t)^{-N / 2} e^{-\left(|\boldsymbol{x}|^{2}+|\boldsymbol{y}|^{2}\right) / 2 t} \operatorname{det}_{1 \leq j, k \leq N}\left[e^{x_{j} y_{k} / t}\right]$, gives

$$
\lim _{|\boldsymbol{x}| \rightarrow 0} p_{N}^{\boldsymbol{\nu}}(t, \boldsymbol{y} \mid \boldsymbol{x})=e^{-t|\boldsymbol{\nu}|^{2} / 2} \operatorname{det}_{1 \leq j, k \leq N}\left[e^{\nu_{j} y_{k}}\right](2 \pi t)^{-N / 2} e^{-|\boldsymbol{y}|^{2} / 2 t} \frac{h_{N}(\boldsymbol{y} / t)}{h_{N}(\boldsymbol{\nu})},
$$

which is equal to (1.21).

\section{B On the O'Connell process with drift $\nu$}

Since the formula (1.17) is not found in [30], here we give explanation for it and then prove (1.20). Note that the derivation of (1.17) with $\boldsymbol{\nu}=0$ was given in $[15,16]$.

For $a>0, \boldsymbol{\nu} \in \mathbb{R}^{N}$, we consider the following partial differential equation

$$
\left[\frac{\partial}{\partial t}+\mathcal{H}_{N}-\frac{1}{a} \boldsymbol{\nu} \cdot \nabla\right] u_{a}^{\boldsymbol{\nu}}(t, \boldsymbol{x})=0, \quad \boldsymbol{x} \in \mathbb{R}^{N}, \quad t \in[0, \infty),
$$

where

$$
\mathcal{H}_{N}=-\frac{1}{2} \Delta+\frac{1}{a^{2}} \sum_{j=1}^{N-1} e^{-\left(x_{j+1}-x_{j}\right) / a}
$$

is identified with the Hamiltonian of an open quantum Toda lattice [30]. Assume that $\boldsymbol{x}, \boldsymbol{\nu} \in \mathbb{W}_{N}$. Then by the Feynman-Kac formula (see, for instance, [13]) the stationary 
solution $u_{a}^{\boldsymbol{\nu}}(\boldsymbol{x})$ of (B.1) with (B.2), which is uniquely determined by imposing the condition $\lim _{|\boldsymbol{x}| \rightarrow \infty, \boldsymbol{x} \in \mathbb{W}_{N}} u_{a}^{\boldsymbol{\nu}}(\boldsymbol{x})=1, \boldsymbol{\nu} \in \mathbb{W}_{N}$, is given by

$$
u_{a}^{\boldsymbol{\nu}}(\boldsymbol{x})=\mathbf{E}^{\boldsymbol{x}}\left[\exp \left(-\frac{1}{a^{2}} \sum_{j=1}^{N-1} \int_{0}^{\infty} e^{-\left\{\widehat{B}_{j+1}(s)-\widehat{B}_{j}(s)\right\} / a} d s\right)\right],
$$

where $\mathbf{E}^{\boldsymbol{x}}[\cdot]$ denotes the expectation with respect to the Brownian motion (1.5) with drift $\boldsymbol{\nu} \in \mathbb{W}_{N}$ and starting from $\boldsymbol{x} \in \mathbb{W}_{N}$. We note that for $0 \leq T<\infty$

$$
\mathcal{N}_{N}^{\boldsymbol{\nu}, a}(T, \boldsymbol{x})=\mathbf{E}^{\boldsymbol{x}}\left[\exp \left(-\frac{1}{a^{2}} \sum_{j=1}^{N-1} \int_{0}^{T} e^{-\left\{\widehat{B}_{j+1}(s)-\widehat{B}_{j}(s)\right\} / a} d s\right)\right]
$$

expresses the probability that, in the mutually killing $N$-particle system with the killing term $-\left(1 / a^{2}\right) \sum_{j=1}^{N-1} e^{-\left(x_{j+1}-x_{j}\right) / a}[16]$, all $N$ Brownian particles with drifts $\left\{\widehat{B}_{j}(t)\right\}_{j=1}^{N}$ starting from $\boldsymbol{x} \in \mathbb{W}_{N}$ survive up to time $T$ and that (B.3) is its long-term limit, $\lim _{T \rightarrow \infty} \mathcal{N}_{N}^{\boldsymbol{\nu}, a}(T, \boldsymbol{x})$, $\boldsymbol{x}, \boldsymbol{\nu} \in \mathbb{W}_{N}$. On the other hand, the class-one Whittaker function $\psi_{\boldsymbol{\nu}}^{(N)}(\boldsymbol{x})$ given by $(1.15)$ is an eigenfunction of the Hamiltonian (B.2) with the eigenvalue $-\sum_{j=1}^{N} \nu_{j}^{2} / 2, \boldsymbol{\nu} \in \mathbb{C}^{N}$. By the method of separation of variables, we can show that $u_{a}(\boldsymbol{x})$ is also expressed by using $\psi_{\boldsymbol{\nu}}^{(N)}(\boldsymbol{x} / a)$. Then the equality

$$
\lim _{T \rightarrow \infty} \mathcal{N}_{N}^{\boldsymbol{\nu}, a}(T, \boldsymbol{x})=c_{N}(\boldsymbol{\nu}) e^{-\boldsymbol{\nu} \cdot \boldsymbol{x} / a} \psi_{\boldsymbol{\nu}}^{(N)}(\boldsymbol{x} / a), \quad \boldsymbol{x}, \boldsymbol{\nu} \in \mathbb{W}_{N}
$$

is established, where $c_{N}(\boldsymbol{\nu})=\prod_{1<j<k<N}\left\{\sin \pi\left(\nu_{k}-\nu_{j}\right)\right\} / \pi[31]$.

We find that $Q_{N}^{a}(t, \boldsymbol{y} \mid \boldsymbol{x})$ given by (1.18) solves (B.1) with $\boldsymbol{\nu}=0$ and $Q_{N}^{a}(0, \boldsymbol{y} \mid \boldsymbol{x})=$ $\delta(\boldsymbol{x}-\boldsymbol{y})$, and then we can regard it as a transition probability density of the mutually killing Brownian motions with duration $t \in[0, \infty)$ from $\boldsymbol{x} \in \mathbb{R}^{N}$ to $\boldsymbol{y} \in \mathbb{R}^{N}$, preserving the number of particles $[15,16]$. Then the transition probability density of the mutually killing Brownian motions with drift $\boldsymbol{\nu}$ conditioned that all particle survive is given by

$$
P_{N}^{\boldsymbol{\nu}, a}(t, \boldsymbol{y} \mid \boldsymbol{x})=\lim _{T \rightarrow \infty} \frac{\mathcal{N}_{N}^{\boldsymbol{\nu}, a}(T-t, \boldsymbol{y})}{\mathcal{N}_{N}^{\boldsymbol{\nu}, a}(T, \boldsymbol{x})} Q_{N}^{\boldsymbol{\nu}, a}(t, \boldsymbol{y} \mid \boldsymbol{x})
$$

with the drift transform of (1.18) with parameter $a>0$

$$
Q_{N}^{\boldsymbol{\nu}, a}(t, \boldsymbol{y} \mid \boldsymbol{x})=\exp \left\{-\frac{t|\boldsymbol{\nu}|^{2}}{2 a^{2}}+\frac{\boldsymbol{\nu}}{a} \cdot(\boldsymbol{y}-\boldsymbol{x})\right\} Q_{N}^{a}(t, \boldsymbol{y} \mid \boldsymbol{x}) .
$$

If $\boldsymbol{x}, \boldsymbol{y}, \boldsymbol{\nu} \in \mathbb{W}_{N}$, by (B.5), (B.6) is equal to (1.17), which should solve (1.16) with the initial condition $P_{N}^{\boldsymbol{\nu}, a}(0, \boldsymbol{y} \mid \boldsymbol{x})=\delta(\boldsymbol{x}-\boldsymbol{y})$.

The class-one Whittaker function has the alternating sum formula [1],

$$
\psi_{\boldsymbol{\nu}}^{(N)}(\boldsymbol{x})=c_{N}(\boldsymbol{\nu})^{-1} \sum_{\sigma \in \mathcal{S}_{N}} \operatorname{sgn}(\sigma) m^{(N)}(\boldsymbol{x}, \sigma(\boldsymbol{\nu})),
$$


where $\sigma(\boldsymbol{\nu})=\left(\nu_{\sigma(1)}, \ldots, \nu_{\sigma(N)}\right)$ for each permutation $\sigma \in \mathcal{S}_{N}$. Here $m^{(N)}(\boldsymbol{x}, \boldsymbol{\nu})$ is the fundamental Whittaker function, which is normalized here as $\lim _{a \rightarrow 0} m^{(N)}(\boldsymbol{x} / a, a \boldsymbol{\nu})=e^{\boldsymbol{\nu} \cdot \boldsymbol{x}}$ for $\boldsymbol{x} \in \mathbb{W}_{N}$. Since $c_{N}(a \boldsymbol{\nu}) \simeq a^{N(N-1) / 2} h_{N}(\boldsymbol{\nu})$ as $a \rightarrow 0$, we have

$$
\lim _{a \rightarrow 0} a^{N(N-1) / 2} \psi_{a \boldsymbol{\nu}}^{(N)}(\boldsymbol{x} / a)=\frac{\operatorname{det}_{1 \leq j, k \leq N}\left[e^{\nu_{j} x_{k}}\right]}{h_{N}(\boldsymbol{\nu})} \text { for } \boldsymbol{x}, \boldsymbol{\nu} \in \mathbb{W}_{N} .
$$

Moreover, the density of Sklyanin measure (1.19) has the asymptotics in $a \rightarrow 0$ as $s_{N}(a \boldsymbol{k}) \simeq$ $a^{N(N-1)}\left(h_{N}(\boldsymbol{k})\right)^{2} /\left\{(2 \pi)^{N} N !\right\}$, and thus (1.18) gives [16]

$$
\begin{aligned}
\lim _{a \rightarrow 0} Q_{N}^{a}(t, \boldsymbol{y} \mid \boldsymbol{x}) & =\frac{1}{(2 \pi)^{N} N !} \int_{\mathbb{R}^{N}} e^{-t|\boldsymbol{k}|^{2} / 2} \operatorname{det}_{1 \leq j, \ell \leq N}\left[e^{i k_{j} x_{\ell}}\right] \operatorname{det}_{1 \leq j, \ell \leq N}\left[e^{-i k_{j} y_{\ell}}\right] d \boldsymbol{k} \\
& =q_{N}(t, \boldsymbol{y} \mid \boldsymbol{x}), \quad \boldsymbol{x}, \boldsymbol{y} \in \mathbb{W}_{N}, \quad t \in[0, \infty) .
\end{aligned}
$$

Then (1.20) is concluded. Note that the first equality in (B.10) can be interpreted in terms of the Slater determinants used in quantum mechanics [33].

Acknowledgements The present author would like to thank T. Imamura and P. Graczyk for useful discussion on diffusion processes with drifts. A part of the present work was done during the participation of the author in the EPSRC Symposium Workshop on "Interacting particle systems, growth models and random matrices" at the university of Warwick (19-23 March 2012). The author thanks N. O'Connell, J. Ortmann, and J. Warren for invitation to the workshop. This work is supported in part by the Grant-in-Aid for Scientific Research (C) (No.21540397) of Japan Society for the Promotion of Science.

\section{References}

[1] Baudoin, F., O'Connell, N.: Exponential functionals of Brownian motion and class-one Whittaker functions. Ann. Inst. H. Poincaré, B 47, 1096-1120 (2011)

[2] Biane, P.: Permutation model for semi-circular systems and quantum random walks. Pacific J. Math. 171, 373-387 (1995)

[3] Biane, P., Bougerol, P., O'Connell, N.: Littelmann paths and Brownian paths. Duke Math. J. 130, 127-167 (2005)

[4] Biane, P., Bougerol, P., O'Connell, N.: Continuous crystal and Duistermaat-Heckman measure for Coxeter groups. Adv. Math. 221, 1522-1583 (2009)

[5] Borodin, A., Corwin, I.: Macdonald processes. arXiv:math.PR/1111.4408

[6] Corwin, I., O'Connell, N., Seppäläinen, T., Zygouras, N.: Tropical combinatorics and Whittaker functions. arXiv:math.PR/1110.3489

[7] Dyson, F. J.: A Brownian-motion model for the eigenvalues of a random matrix. J. Math. Phys. 3, 1191-1198 (1962) 
[8] Forrester, P. J.: Log-gases and Random Matrices. London Mathematical Society Monographs, Princeton University Press, Princeton (2010)

[9] Fulton, W., Harris, J.: Representation Theory, A First Course. Springer, New York (1991)

[10] Grabiner, D. J.: Brownian motion in a Weyl chamber, non-colliding particles, and random matrices. Ann. Inst. Henri Poincaré, Probab. Stat. 35, 177-204 (1999)

[11] Johansson, K.: Determinantal processes with number variance saturation. Commun. Math. Phys. 252, 111-148 (2004)

[12] Jones, L., O'Connell, N.: Weyl chambers, symmetric spaces and number variance saturation. Alea 2, 91-118 (2006)

[13] Karatzas, I., Shreve, S. E.: Brownian Motion and Stochastic Calculus. 2nd edn. Springer, (1991)

[14] Karlin, S., McGregor, J.: Coincidence probabilities. Pacific J. Math. 9, 1141-1164 (1959)

[15] Katori, M.: O'Connell's process as a vicious Brownian motion. Phys. Rev. E 84, 061144 (2011)

[16] Katori, M.: Survival probability of mutually killing Brownian motion and the O'Connell process. J. Stat. Phys. 147, 206-223 (2012)

[17] Katori, M.: System of complex Brownian motions associated with the O'Connell process. arXiv:math.PR/1206.2185

[18] Katori, M., Tanemura, H.: Scaling limit of vicious walks and two-matrix model. Phys. Rev. E 66, 011105 (2002)

[19] Katori, M., Tanemura, H.: Symmetry of matrix-valued stochastic processes and noncolliding diffusion particle systems. J. Math. Phys. 45, 3058-3085 (2004)

[20] Katori, M., Tanemura, H.: Noncolliding Brownian motion and determinantal processes. J. Stat. Phys. 129, 1233-1277 (2007)

[21] Katori, M., Tanemura, H.: Zeros of Airy function and relaxation process. J. Stat. Phys. 136, 1177-1204 (2009)

[22] Katori, M., Tanemura, H.: Non-equilibrium dynamics of Dyson's model with an infinite number of particles. Commun. Math. Phys. 293, 469-497 (2010)

[23] Katori, M., Tanemura, H.: Noncolliding squared Bessel processes. J. Stat. Phys. 142, 592-615 (2011)

[24] Katori, M., Tanemura, H.: Noncolliding processes, matrix-valued processes and determinantal processes. Sugaku Expositions 24, 263-289 (2011); arXiv:math.PR/1005.0533 
[25] Katori, M., Tanemura, H.: Complex Brownian motion representation of the Dyson model. arXiv:math.PR/1008.2821

[26] Levin, B. Ya.: Lectures on Entire Functions. Translations of Mathematical Monographs, 150, Amer. Math. Soc, Providence R. I. (1996)

[27] Matsumoto, H., Yor, M.: An analogue of Pitman's $2 M-X$ theorem for exponential Wiener functionals, Part I: A time-inversion approach. Nagoya Math. J. 159, 125-166 (2000)

[28] Matsumoto, H., Yor, M.: Exponential functionals of Brownian motion I: Probability laws at fixed time. Probab. Surveys 2, 312-347 (2005)

[29] Mehta, M. L.: Random Matrices. 3rd edn. Elsevier, Amsterdam (2004)

[30] O'Connell, N.: Directed polymers and the quantum Toda lattice. Ann. Probab.40, 437-458 (2012)

[31] O'Connell, N.: Whittaker functions and related stochastic processes. arXiv:math.PR/1201.4849

[32] Revuz, D., Yor, M.: Continuous Martingales and Brownian Motion. 3rd edn. Springer, Now York (1998)

[33] Schehr, G., Majumdar, S. N., Comtet, A., Randon-Furling, J.: Exact distribution of the maximal height of $p$ vicious walkers. Phys. Rev. Lett. 101, 150601/1-4 (2008)

[34] Shirai, T., Takahashi, Y.: Random point fields associated with certain Fredholm determinants I: fermion, Poisson and boson point process. J. Funct. Anal. 205, 414-463 (2003)

[35] Soshnikov, A. : Determinantal random point fields. Russian Math. Surveys 55, 923-975 (2000) 